\title{
Deep Brain Stimulation of the Cuneiform Nucleus for Levodopa-Resistant Freezing of Gait in Parkinson's Disease: Study Protocol for a Prospective, Pilot Trial
}

\section{Stephano Chang}

University of Miami School of Medicine https://orcid.org/0000-0002-6168-4341

\section{lahn Cajigas}

University of Miami School of Medicine

James D. Guest

University of Miami School of Medicine

\section{Brian R. Noga}

University of Miami School of Medicine

Corneliu C. Luca

University of Miami

Jonathan R. Jagid ( $\sim$ jjagid@med.miami.edu )

The Miami Project to Cure Paralysis, Miami, FL USA 2Department of Neurological Surgery, University of Miami Miller School of Medicine, Miami, FL USA

\section{Study Protocol}

Keywords: Freezing of gait (FOG), gait dysfunction, Parkinson's Disease, mesencephalic locomotor region (MLR), cuneiform nucleus $(\mathrm{CnF})$, pedunculopontine nucleus (PPN)

Posted Date: August 20th, 2020

DOI: https://doi.org/10.21203/rs.3.rs-60496/v1

License: (c) (i) This work is licensed under a Creative Commons Attribution 4.0 International License. Read Full License

Version of Record: A version of this preprint was published at Pilot and Feasibility Studies on June 2nd, 2021. See the published version at https://doi.org/10.1186/s40814-021-00855-7. 


\section{Abstract}

Background: Freezing of gait (FOG) is a particularly debilitating motor deficit seen in a subset of Parkinson's Disease (PD) patients that is poorly responsive to standard levodopa therapy or deep brain stimulation (DBS) of established PD targets such as the subthalamic nucleus and the globus pallidus interna. The proposal of a DBS target in the midbrain, known as the pedunculopontine nucleus (PPN) to address FOG was based on its observed pathology in PD and its hypothesized involvement in locomotor control as a part of the mesencephalic locomotor region, a functionally defined area of the midbrain that elicits locomotion in both intact animals and decerebrate animal preparations with electrical stimulation. Initial reports of PPN DBS were met with much enthusiasm; however, subsequent studies produced mixed results, and recent meta-analysis results have been far less convincing than initially expected. A closer review of the extensive MLR preclinical literature, including recent optogenetics studies, strongly suggests that the closely related cuneiform nucleus (CnF), just dorsal to the PPN, may be a superior target to promote gait initiation.

Methods: We will conduct a prospective, open-label, single-arm pilot study to assess safety and feasibility of $\mathrm{CnF}$ DBS in PD patients with levodopa-refractory FOG. Four patients will receive CnF DBS and have gait assessments with and without DBS during a 6-month follow up.

Discussion: This paper presents the study design and rationale for a pilot study investigating a novel DBS target for gait dysfunction, including targeting considerations. This pilot study is intended to support future larger scale clinical trials investigating this target.

Trial Registration: Clinicaltrials.gov Identifier: NCT04218526 (registered January 6, 2020), https://www.clinicaltrials.gov/ct2/show/NCT04218526

\section{Background And Rationale}

\section{Refractory freezing of gait in Parkinson's disease}

Gait dysfunction is a common and heterogeneous feature of Parkinson's disease (PD), significantly impacting patients' quality of life and increasing their risk for falls (1). Common characteristics of parkinsonian gait include decreased stride length, reduced arm swing amplitude, and a flexed and stiffened trunk posture (2). Some of these aspects of gait are improved with dopaminergic therapy or deep brain stimulation (DBS) of conventional targets such as the subthalamic nucleus (STN) or globus pallidus interna (GPi) (3). Other deficits, such as postural instability and freezing of gait (FOG), may not respond or even worsen (4).

FOG is considered the most disabling of these gait deficits, and is described as the transient and episodic failure to move forwards, despite intending to walk (5). Patients describe feeling as if their feet are "glued" to the floor, and these episodes result in significantly decreased quality of life and contribute to falls and their related morbidities (6-8). FOG is triggered in situations that highlight both cognitive and sensory influences on gait: during attempts to initiate stepping or turning, when navigating narrow corridors and obstacles, and when the patient is distracted or under duress $(9,10)$. Conversely, these episodes frequently improve or resolve with the help of auditory or visual cues, such as a metronome set to a step cadence, or lines marked on the floor set to a desired stride length (11).

The pathophysiology of FOG is incompletely understood, but observations that it can be refractory to dopaminergic therapy suggest that it involves both dopaminergic and non-dopaminergic mechanisms (12). FOG is 
associated with advanced PD (13), though it can present early (11). While some controversy remains as to whether severe gait difficulties represent a distinct spectrum of the disease or merely a more advanced stage of the disease (14), many clinicians distinguish between tremor dominant (TD) and postural instability and gait difficulty dominant (PIGD) subtypes of PD, both in terms of their clinical prognoses as well as their management (15). FOG is predominantly seen in the PIGD subtype and has an inconsistent response to levodopa or DBS. The development of an effective treatment for FOG remains a priority in the field.

\section{Rationale for DBS of the mesencephalic locomotor region}

The mesencephalic locomotor region (MLR) is a physiologically defined area in the midbrain tegmentum, where electrical stimulation was found to initiate locomotion in cats $(16,17)$. Since its discovery in 1966 , the MLR has been identified in multiple species, as a phylogenetically preserved node in the supraspinal locomotor network (1823), with studies suggesting therapeutic potential in animal models of gait disorders (24-26). Electrophysiological and functional imaging evidence also supported its existence in humans (27-29), encouraging clinical interest in the region as a DBS target to promote gait. Specifically, this was thought to be a potential treatment for patients with the PIGD subtype of PD (15) and FOG that did not respond to levodopa therapy or conventional DBS of the STN or GPi. (30-32).

Over the past 15 years, several centers have reported on DBS of the pedunculopontine nucleus (PPN), a putative anatomical component of the mesencephalic locomotor region (MLR), for postural instability and gait dysfunction in PD (33). Despite initially promising case reports $(34,35)$, the efficacy of this therapy has since been called into dispute through the results of double-blinded studies and meta-analyses (36-39).

Several reasons have been posited to explain this lack of efficacy, including potential species differences in MLR function, and the degeneration of MLR neurons in PD (40-42). A recent clinical study of DBS assessed FOG outcomes using a responder analysis. Of the reported subjects, there was a "good responder" cluster with near resolution of percent time spent in FOG with DBS on compared to off (34.1 $\pm 14 \%$ vs. $2.7 \pm 2.6 \%$ ) (43). The best responders had active electrodes in the dorsal part of the MLR in the cuneiform nucleus (CnF) rather than the PPN (Figure 1) (43). This aligns with recent optogenetic studies in rodents, which showed that stimulation of glutamatergic neurons in the CnF effectively and specifically caused locomotor initiation $(44,45)$ (For review see (46). It also agrees with preclinical electrophysiological studies in the cat, where stimulation of low threshold sites within the MLR primarily showed CnF neuron activation (47). The importance of electrode targeting on efficacy in this region is reinforced by computer modeling studies, demonstrating that targeting errors of $1 \mathrm{~mm}$ can significantly decrease target activation selectivity (48).

Thus, this pilot study is designed to test the safety, feasibility, and preliminary efficacy of CnF DBS in alleviating FOG. Through this and future larger scale studies, we ultimately aim to explore the hypothesis that the $\mathrm{CnF}$ represents the neuroanatomic basis of the MLR, and that suboptimal targeting may have played a role in the equivocal results of prior PPN DBS studies. We hope to confirm the findings in Goetz et al. (43), which suggest that minute changes in DBS target location in this area have significant impacts on clinical outcome, and determine if CnF DBS may be a viable therapy for FOG and other gait dysfunctions.

\section{Methods/design}

\section{Study design and overview}


This study is a prospective, non-randomized, single-arm, open-label, pilot clinical trial designed to evaluate the safety and feasibility of delivering DBS to the $\mathrm{CnF}$ to alleviate freezing of gait in PD patients with severe, levodoparesistant gait freezing. This study was approved by the University of Miami Human Subject Research Office (UM HSRO) and the US Food and Drug Administration with an Investigational Device Exemption as a phase I trial. The trial is registered in ClinicalTrials.gov: (NCT04218526). The study outline is shown in Figure 2.

Suitable eligible patients will be screened for study participation and begin the consenting process, where the study objectives and the risks and benefits of participating are explained to them. Informed consent forms for the study will be filled out by subjects to confirm enrollment and they will undergo a baseline assessment, including a thorough neurological and neuropsychiatric examination, as well as standardized subjective and objective gait assessments. The Columbia Suicide Severity Rating Scale is included during screening given the known risk of suicidality with DBS for PD (49). After enrolment and baseline evaluation, subjects will be consented for surgery prior to bilateral implantation of DBS electrodes in the $\mathrm{CnF}$, with a post-operative CT scan to localize final electrode positions. For ethical reasons, all subjects will receive active stimulation beginning at two weeks post-implantation and will undergo repeated gait assessments in the clinic at 2, 6, 12, and 24 weeks post implantation (Table 1). Gait assessment tests will be performed on medication, with DBS on and off, and assessors will be blinded to whether the DBS is on or off to minimize the risk of bias. A final clinic visit will take place at 25 weeks post implantation to perform a more detailed kinematics and EMG gait assessment, using the Nexus system (Vicon Motion Systems Ltd). At study conclusion, subjects will decide whether they want to continue with stimulation or discontinue stimulation; in both cases they will continue to receive their usual standard care and follow up with their neurologist.

\section{Table 1. Detailed Study Schedule.}




\begin{tabular}{|c|c|c|c|c|c|c|c|c|c|}
\hline \multirow[t]{2}{*}{ Assessment } & Visit 1 & Visit 2 & Visit 3 & Visit 4 & $\begin{array}{l}\text { Visit } \\
5\end{array}$ & $\begin{array}{l}\text { Visit } \\
6\end{array}$ & $\begin{array}{l}\text { Visit } \\
7\end{array}$ & $\begin{array}{l}\text { Visit } \\
8\end{array}$ & $\begin{array}{l}\text { Visit } \\
9\end{array}$ \\
\hline & Screen & $\begin{array}{l}\text { Baseline } \\
\text { assessment }\end{array}$ & $\begin{array}{l}\text { Surgical } \\
\text { screening }\end{array}$ & Surgery & $\begin{array}{l}2- \\
\text { week } \\
\text { F/U }\end{array}$ & $\begin{array}{l}\text { 6- } \\
\text { week } \\
\text { F/U }\end{array}$ & $\begin{array}{l}12- \\
\text { week } \\
\text { F/U }\end{array}$ & $\begin{array}{l}\text { 24- } \\
\text { week } \\
\text { F/U }\end{array}$ & $\begin{array}{l}\text { 25- } \\
\text { week } \\
\text { F/U }\end{array}$ \\
\hline Screening ICF & $\mathrm{x}$ & & & & & & & & \\
\hline $\begin{array}{l}\text { Preop Work up } \\
\text { (CBC, CMP, } \beta- \\
\text { hCG,INR/PTT/PT, } \\
\text { U/A, EKG, CXR) }\end{array}$ & $x$ & & $x$ & & & & & & \\
\hline $\begin{array}{l}\text { Neuropsychiatric } \\
\text { evaluation } \\
\text { (including MDRS } \\
\text { and BDI) }\end{array}$ & $x$ & $x$ & $x$ & & $x$ & $x$ & $x$ & $x$ & \\
\hline $\begin{array}{l}\text { Columbia } \\
\text { Suicide Severity } \\
\text { Rating Scale }\end{array}$ & $x$ & $x$ & $x$ & & $x$ & $x$ & $x$ & $x$ & \\
\hline Gait Assessment & $x$ & $x$ & & & $x$ & $x$ & $x$ & $x$ & \\
\hline MDS-UPDRS & $\mathrm{x}$ & $\mathrm{x}$ & & & $x$ & $\mathrm{x}$ & $\mathrm{x}$ & $\mathrm{x}$ & \\
\hline $\begin{array}{l}\text { FOG } \\
\text { Questionnaire }\end{array}$ & & $x$ & & & $x$ & $x$ & $x$ & $x$ & \\
\hline $\begin{array}{l}\text { Timed Up and Go } \\
\text { test }\end{array}$ & & $x$ & & & $x$ & $x$ & $x$ & $x$ & \\
\hline Velocity test & & $\mathrm{x}$ & & & $x$ & $x$ & $\mathrm{x}$ & $x$ & \\
\hline Variability test & & $x$ & & & $x$ & $x$ & $\mathrm{x}$ & $\mathrm{x}$ & \\
\hline PDQ-39 & & $x$ & $x$ & & $x$ & $x$ & $x$ & $x$ & \\
\hline PDQL & & $\mathrm{x}$ & $x$ & & $x$ & $\mathrm{x}$ & $\mathrm{x}$ & $\mathrm{x}$ & \\
\hline Brief Pain History & & $x$ & $x$ & & $x$ & $x$ & $x$ & $x$ & \\
\hline Surgical ICF & & & $x$ & & & & & & \\
\hline MRI & & & $x$ & & & & & & \\
\hline $\begin{array}{l}\text { Device } \\
\text { Implantation }\end{array}$ & & & & $x$ & & & & & \\
\hline CT scan & & & & $\mathrm{x}$ & & & & & \\
\hline EMG analysis & & & & $x$ & & & & & $x$ \\
\hline Programming & & & & & $x$ & $x$ & $x$ & $x$ & $x$ \\
\hline Gait kinematics & & & & & & & & & $x$ \\
\hline
\end{tabular}

BDI Beck Depression Inventory, $\beta$-hCG beta human chorionic gonadotropin, CBC complete blood count, CMP comprehensive metabolic panel, CT computed tomography, CXR chest x-ray, EKG electrocardiogram, EMG 
electromyogram, FOG freezing of gait, F/U follow up, INR international normalized ratio, MDRS Mattis Dementia Rating Scale, MDS-UPDRS Movement Disorder Society Unified Parkinson's Disease Rating Scale, MRI magnetic resonance imaging, PDQ-39 Parkinson's Disease Questionnaire-39, PDQL Parkinson's Disease Quality of Life questionnaire, PT prothrombin time, PTT partial thromboplastin time, U/A urinalysis,

*Pre-op labs are viable for 30 days before surgery. After 30 days they must be repeated before surgical procedure

\section{Participants}

We are actively screening and recruiting patients for this study from the Movement Disorders Clinic at the University of Miami Hospital. As this is a pilot study, we plan to enroll 4 patients with PD with levodopa-resistant FOG. A movement disorder neurologist will assess the eligibility of a patient based on the inclusion and exclusion criteria are detailed below.

\section{Inclusion criteria}

- Age 40-75

- PD stage 3 with good response to levodopa (defined as greater than $20 \%$ improvement in MDS-UPDRS score), except for severe gait disorder

- PD stage 3 with severe gait dysfunction and predominant axial symptoms defined as: TD/PIGD ratio $\leq 90$ (mean value of MDS-UPDRS items 2.10, 3.15a, 3.15b, 3.16a, 3.16b, 3.17a, 3.17b, 3.17c, 3.17d, 3.17e, and 3.18 divided by the mean value of MDS-UPDRS items 2.12, 2.13,3.10,3.11, and 3.12)(15) and FOGQ score> 12

- FOG refractory to levodopa>600 mg

- Minimal tremor, bradykinesia, and rigidity, or well controlled with levodopa.

- Agrees to full 6-month study participation

\section{Exclusion criteria}

- Individuals with major executive dysfunction, dementia (Mattis Dementia Rating Scale-2 score $\leq 130$ ), depression (Beck Depression Inventory II $\geq 25$ ), or other neurocognitive impairments

- Presence of major medical co-morbidities and other surgical contra-indications

- Individuals requiring diathermy, transcranial magnetic stimulation, or electroconvulsive therapy

- Individuals with prior intracranial surgery

- Individuals with non-MRI compatible metallic implants in their head or active implantable devices anywhere in the body

- Individuals who are pregnant, breastfeeding, or the desire to become pregnant during the study

- Individuals on investigational drugs or any other intervention (not part of the guidelines for management of PD) known to have a potential impact on outcome

\section{Assessments}

The following assessments will be completed to screen for study/surgical eligibility:

1. History and physical exam, including a neurological and clinical gait assessment

2. Blood pressure and postural drop 
3. Pre-operative lab work, including 12-lead electrocardiogram and chest x-ray

4. Neuropsychiatric evaluation, including the Mattis Dementia Rating Scale-2, Beck Depression Inventory II, and the Columbia Suicide Severity Rating Scale

The following assessments will be completed prior to DBS implantation as well as at each clinic visit postimplantation:

1. MDS-UPDRS

2. FOG Questionnaire

3. Timed Up and Go (TUG) test

4. Clinical gait assessment measuring:

a. Stride Length

b. Velocity

c. Gait variability measured over a 2-minute walk

5. The Parkinson's Disease Questionnaire (PDQ-39) and the Parkinson's Disease Quality of Life Questionnaire (PDQL)

6. Columbia Suicide Severity Rating Scale

7. Neuropathic pain inventory and history

8. Blood pressure and postural drop assessed in the off and on DBS stimulation state.

At study conclusion, patients will undergo a detailed kinematics gait assessment using the Nexus system (Vicon Motion Systems Ltd) and surface EMG recordings in the lower limbs.

\section{Device implantation}

The Vercise $^{\mathrm{TM}}$ (Boston Scientific Corporation) DBS System and Cartesia ${ }^{\mathrm{TM}}$ (Boston Scientific Corporation) directional leads will be used in this study. Implantation of electrodes and generator will be performed in one procedure, as previously described at our institution,(50) but targeting the CnF. A pre-operative 3T MRI will be obtained prior to surgery. Subjects will be admitted on the day of surgery, and under intravenous sedation, a CRW frame will be placed and a CT obtained. The CT and MRI will be merged to obtain frame-based coordinates for the $\mathrm{CnF}$. The default CnF coordinates will be calculated based on MRI brainstem landmarks to target brainstem normalized coordinates of $(0.50,0.25,0)(43)$, and diffusion tractography will be used to ensure that our target is within the area demarcated by the medial lemniscus, superior cerebellar peduncle, and central tegmental tract. Trajectory planning will be performed to avoid vessels and ependymal and pial surfaces. Blood pressure and heart rate will be monitored by arterial line, and systolic blood pressure will be maintained between $90-120 \mathrm{mmHg}$ during electrode insertions to reduce the risk of hemorrhage. The DBS leads will be placed with intraoperative test stimulations to assess efficacy and rule out potential side-effects that may warrant repositioning of the lead (Figure 1C). Intraoperative lower-extremity EMG will be used to assess for muscle activation to assist with targeting. The use of an intraoperative 0 -arm (Medtronic) spin to provide real-time estimates of lead trajectory and location will be an option if there are any concerns about targeting or off-target stimulation effects. After implantation, patients will be fully anesthesized to undergo subcutaneous implantation of the generator in the chest to complete the procedure. Patients will be admitted to a post-surgical unit overnight for monitoring, including blood pressure and vital signs. 


\section{DBS programming}

Intraoperative stimulation parameters will be used to guide initial programming. Programming sessions will have heart rate, blood pressure, respiratory rate, and blood oxygenation saturation monitoring to mitigate the risk of adverse cardiorespiratory events related to stimulation. Additionally, low frequencies will initially be explored with patients until an adequate stimulation amplitude is found where gait initiation is under volitional control. Stimulation amplitude will then be increased until an adequate gait speed is achieved. Based on other studies, cyclic stimulation with continuous daily stimulation and night arrests will be the default protocol to avoid habituation and waning of effects $(43,51)$. Current steering will be used to increase the therapeutic window and maximize chances of therapeutic benefit.

\section{Efficacy outcomes}

Outcomes of neurological and functional status, gait, and quality-of-life are collected at several time points during the study (Table 1).

\section{Primary outcomes}

1. Percent change in gait velocity with and without CnF DBS

2. Percent change in Movement Disorders Society Unified Parkinson's Disease Rating Scale (MDS-UPDRS) Part III across study visits relative to pre-operative baseline evaluation.

\section{Secondary outcomes}

1. Percent change in FOG Questionnaire (FOGQ), Parkinson's Disease Questionnaire (PDQ-39), and the Parkinson's Disease Quality of Life Questionnaire (PDQL)

2. Percent change in muscle electromyogram (EMG) amplitude during gait testing at study conclusion between CnF DBS on and off conditions

\section{Kinematic and EMG Evaluation of Gait}

Lower-limb gait kinematics and EMG will be evaluated with and without DBS in study subjects $(n=4)$. Subjects will be fitted with fifteen reflective markers that will be tracked at $100 \mathrm{~Hz}$ with a 10 -camera Nexus system (Vicon Motion Systems Ltd) attached bilaterally to the first and fifth distal phalange of the foot, lateral malleolus, calcaneus, lateral femoral epicondyle, anterior superior iliac spine, as well as the mid-shank and mid-thigh. A reflective marker will also be placed on the lower back region overlying the sacrum. EMG surface electrodes will be placed over the tibialis anterior (TA) as well as the lateral gastrocnemius (LG). Subjects will be asked to walk along a 25-foot path at a constant, regular walking pace both without and then with optimal DBS configuration settings (CnF L on/CnF R on), DBS OFF (CnF L off/CnF R off), DBS RIGHT Only (CnF L off/CnF R on), DBS LEFT only (CnF $\mathrm{L}$ on/CnF R off) repeating each condition twice. Kinematic and EMG signals will be recorded during each trial while the subjects walk for 25 feet and averaged for each test condition.

\section{Safety}

The analyses for safety will be descriptive, focusing on trends for within-subject differences and changes in FOG symptoms or off-target side-effects during the duration of the study. All adverse events (AEs) will be listed and 
their incidence compared to historical controls. The primary safety analysis will be conducted on all patient data when all 4 participants have completed the study.

\section{Handling of missing data}

Because of the 25-week follow-up, efforts will be made to minimize the number of participants lost to follow-up by developing good rapport, making the participant feel comfortable with the research staff and having regular correspondence between assessments. To maintain contact and continued willingness for study participation at each visit participants will provide their current address and phone number and e-mail address as well as contact information for at least two individuals who live outside of their household yet are likely to know their whereabouts. Contact information and contact history will be entered into an electronic database allowing regular review and update.

Although every attempt will be made to avoid missing outcome data, missing data is anticipated with any longitudinal study. The missing data can be defined as intermittent or dropout. The impact of missing data will be examined by comparing means and standard deviations for participants that have and do not have the relevant missed visit data. Where appropriate, imputation of extreme values will be used to demonstrate negligible impact of the missing data. The reason for missing data will also be recorded and fully examined to confirm this assumption, and to minimize this occurrence in any future confirmatory trials. Where appropriate, non-negligible missing data will be imputed using regression methods.

\section{Data analysis}

The primary goal of this pilot study is to assess the safety and feasibility of delivering DBS to the CnF in PD patients with refractory FOG, and thus will only involve 4 subjects. We believe this number of subjects will provide adequate data on safety and feasibility for a larger study. However, assessment of trend toward efficacy will be performed for the primary and secondary outcomes. One-way ANOVA will be used to compare percent improvement in gait velocity for the four different DBS configurations ( $L \mathrm{CnF}$ off/R CnF off; $L \mathrm{CnF}$ off/R $\mathrm{CnF}$ on; $\mathrm{L}$ $\mathrm{CnF}$ on/R CnF off; L CnF on/R CnF on) at each clinic visit, with the Tukey Honest Significant Difference post-hoc test to compare configurations. The same test will be used to compare changes in EMG amplitude between the four DBS configurations at the final gait assessment at the study's conclusion. One-way ANOVA with repeated measures will be used to compare changes in the UPDRS III, FOG Questionnaire, PDQ-39, and PDQL over the multiple time points of the study.

\section{Data management}

All study documents and files will use an anonymous study identification number to identify subjects and only an appointed Study Coordinator will maintain linkages, to maintain subject confidentiality. Additionally, locally stored computer data will be password protected and located within our institution-based firewall. Physical study data files including signed consent forms will be stored securely in the principal investigator's (JJ) office within a locked filing cabinet. Subject data will be documented in source documents initially and then recorded on electronic case report forms (CRFs). The Study Coordinator will ensure that data is entered into CRFs within 5 days of data collection and will review the accuracy of entered data by comparison with subjects' medical records within 30 days. Potential discrepancies will be flagged and checked and corrected as necessary by study investigators. 
Only personnel listed on study protocols will have access to study data, though study data that could medically benefit subjects will be shared with them. There will be periodic audits (at least two during the study) of the data by university's Office of Research Compliance and Quality Assurance (RCQA) auditors to ensure compliance with the FDA's Good Clinical Practices and ICH-E6. The Principal Investigator will submit all de-identified CRFs to the Study Sponsors and the FDA throughout and at the completion of the study, including any incomplete CRFs and CRFs of those who withdraw before study completion.

A Data Safety and Monitoring Board (DSMB) has been established as an independent expert advisory group to assess adverse events (AEs) and evaluate the general integrity and conduct of the study. The DSMB chair will be provided a monthly update on the status of all study subjects. Furthermore, all AEs related to the study will be reported to the DSMB, which will make recommendations as to whether the study should continue without change, be modified, or be terminated early. Pre-determined stoppage rules have also been established to aid with early termination decision making (Table 2). No formal interim analysis of efficacy is planned given the small number of participants.

\section{Table 2. Pre-determined Study Stoppage Rules.}

1 Occurrence of one or more SAEs with unexplained etiology and unsatisfactory resolution

2 Occurrence of one or more persistent and debilitating stimulation-related AEs in the CnF

3 Occurrence of hemorrhage, stroke, or paralysis related to device

4 Occurrence of changes in blood pressure, heart rate, and/or respiratory rate related to device function that occur outside of the clinic and require medical intervention

5 Significant worsening of any symptom of PD, other than transiently, including tremor, bradykinesia, rigidity, or gait that would not otherwise have been expected as part of the natural course of the disease

\section{AE adverse event, CnF Cuneiform nucleus, PD Parkinson's Disease, SAEs serious adverse event}

\section{Discussion}

PD patients with FOG that is refractory to dopaminergic therapy have limited therapeutic options. Our study protocol aims to test the safety, feasibility, and preliminary efficacy of a novel brainstem target to alleviate FOG. Our strategy is based on a careful revisiting of classical animal studies (16), newly emerging optogenetics data (44), as well as a recent clinical study (43), all of which suggest that optimizing the location of DBS within the MLR could significantly improve the effect of DBS on this gait network. While some have pointed to the inconsistent outcomes of previous PPN DBS studies as a reason to abandon this approach altogether (41), that subsets of patients with FOG show significant improvement with DBS in this region suggests that refinement of methodology may be important. Through this and future larger scale studies, we hope to determine if CnF DBS could be an effective therapy for FOG and gait dysfunction in other neurodegenerative disorders.

A strength of this study is the novel use of directional electrodes to deliver DBS to this midbrain region. As a complex area comprised of numerous interleaving fiber tracts and structures, the ability to steer current would be important in optimizing therapeutic effects while mitigating stimulation-induced side effects. Limitations of this study include the small sample size, given the preliminary nature of this study, and the lack of a control cohort. Additionally, as some patients with DBS in this region feel mild paresthesias with stimulation, complete double 
blinding of DBS status during gait assessments may be impossible. Finally, there are limitations related to the studying of FOG itself, including potential heterogeneity of pathobiology, and difficulty in eliciting FOG in the clinical setting. We hope to mitigate these difficulties through our rigorous inclusion/exclusion criteria, and by using known triggers for FOG during gait assessments, if necessary.

\section{Trial status}

Study protocol version 2 was approved March 2, 2020 by the FDA. Recruitment started April 27, 2020 and is expected to be completed by December 31, 2020.

\section{Abbreviations}

AE: Adverse event; CT: Computed tomography; CRF: Case report form; CnF: Cuneiform nucleus; DBS: Deep brain stimulation; DSMB: Data Safety Monitoring Board; EMG: Electromyogram; FOG: Freezing of gait; FTD: Frontotemporal dementia; GPi: Globus pallidus interna; LFP: Local field potential; MDS-UPDRS: Movement Disorder Society Unified Parkinson's Disease Rating Scale; MER: Microelectrode recordings; MLR: Mesencephalic locomotor region; MRI: Magnetic resonance imaging; PAG: Periaqueductal gray; PD: Parkinson's disease; PIGD: Postural instability and gait difficulty; PPN: Pedunculopontine nucleus; STN: Subthalamic nucleus

\section{Declarations}

\section{Ethics approval and consent to participate}

The study protocol was approved by the University of Miami Human Subject Research Office (IRB 20190702) and the FDA (IDE G190164). This study will be conducted in accordance with the ethical principles that have their origin in the Declaration of Helsinki and that are consistent with good clinical practice (GCP) and the applicable regulatory requirements. Trial investigators will obtain written informed consent from each participant before study inclusion. Subjects will be free to withdraw from the study at any time.

\section{Consent for publication}

Results from this study will be presented in peer-reviewed publications and meetings without identifying data. Written consent for publication will be obtained from all participants in the study.

\section{Availability of data and materials}

All data in the study protocol are included in this article and supplementary files. Data from the study are unavailable at the time of publication. Once completed, dissemination of non-identifiable study results will occur through publication in a peer-reviewed journal, presentations, and seminars, irrespective of outcomes. Participants will be able to request a copy of study results from the Principal Investigator.

\section{Competing Interests}

The study is funded in part by Boston Scientific through their Investigator Sponsored Research Award. JJ and CL have consulting agreements with Medtronic Inc, Boston Scientific, Inc, and Abbott Medical. JJ has two funded grants through Medtronic and Boston Scientific. The other authors declare that they have no competing interests. 


\section{Funding}

This study is supported by the Boston Scientific Investigator Sponsored Research Award ISRNMB0018 (contact person Kaoru Lee Adair - kay.adair@bsci.com) and the University of Miami Scientific Awards Committee Pilot Study Grant ITS 001003 (contact person Coleen Atkins - catkins@miami.edu). The funders had no role in the design of the study and collection, analysis, interpretation of data, nor in writing the manuscript.

\section{Authors' contributions}

All authors made significant intellectual contributions and contributed to the conception of this study. JJ is the principal investigator. IC and SC drafted the initially approved grants and IC drafted the FDA IDE and IRB protocol. SC created the first draft of this manuscript, which was reviewed and edited by all other authors. All authors read and approved the final manuscript.

\section{Acknowledgements}

We thank Letitia Fisher for her tireless efforts in organizing the submissions and revisions for grants and applications related to this study.

\section{Authors' contributions}

All authors made significant intellectual contributions and contributed to the conception of this study. JJ is the principal investigator. IC and SC drafted the initially approved grants and IC drafted the FDA IDE and IRB protocol. SC created the first draft of this manuscript, which was reviewed and edited by all other authors. All authors read and approved the final manuscript.

\section{References}

1. Allen NE, Schwarzel AK, Canning CG. Recurrent falls in Parkinson's disease: a systematic review. Parkinson's disease. 2013;2013:906274.

2. Wu T, Hallett M, Chan P. Motor automaticity in Parkinson's disease. Neurobiology of disease. 2015;82:226-34.

3. Collomb-Clerc A, Welter ML. Effects of deep brain stimulation on balance and gait in patients with Parkinson's disease: A systematic neurophysiological review. Neurophysiologie clinique = Clinical neurophysiology. 2015;45(4-5):371-88.

4. Galna B, Lord S, Burn DJ, Rochester L. Progression of gait dysfunction in incident Parkinson's disease: Impact of medication and phenotype. Movement Disorders. 2015;30(3):359-67.

5. Giladi N, Nieuwboer A. Understanding and treating freezing of gait in parkinsonism, proposed working definition, and setting the stage. Mov Disord. 2008;23 Suppl 2:S423-5.

6. Bloem BR, Hausdorff JM, Visser JE, Giladi N. Falls and freezing of gait in Parkinson's disease: a review of two interconnected, episodic phenomena. Movement disorders : official journal of the Movement Disorder Society. 2004;19(8):871-84.

7. Perez-Lloret S, Negre-Pages L, Damier P, Delval A, Derkinderen P, Destee A, et al. Prevalence, determinants, and effect on quality of life of freezing of gait in Parkinson disease. JAMA neurology. 2014;71(7):884-90.

8. Canning CG, Paul SS, Nieuwboer A. Prevention of falls in Parkinson's disease: a review of fall risk factors and the role of physical interventions. Neurodegenerative Disease Management. 2014;4(3):203-21. 
9. Giladi N, McMahon D, Przedborski S, Flaster E, Guillory S, Kostic V, et al. Motor blocks in Parkinson's disease. Neurology. 1992;42(2):333-9.

10. Lamberti P, Armenise S, Castaldo V, de Mari M, lliceto G, Tronci P, et al. Freezing gait in Parkinson's disease. European neurology. 1997;38(4):297-301.

11. Nonnekes J, Snijders AH, Nutt JG, Deuschl G, Giladi N, Bloem BR. Freezing of gait: a practical approach to management. The Lancet Neurology. 2015;14(7):768-78.

12. Santens P. Neuromodulatory procedures for gait disorders in Parkinson's disease. Acta Neurologica Belgica. 2018;118(1):13-9.

13. Macht M, Kaussner Y, Möller JC, Stiasny-Kolster K, Eggert KM, Krüger H-P, et al. Predictors of freezing in Parkinson's disease: A survey of 6,620 patients. Movement Disorders. 2007;22(7):953-6.

14. Lee JW, Song YS, Kim H, Ku BD, Lee WW. Alteration of Tremor Dominant and Postural Instability Gait Difficulty Subtypes During the Progression of Parkinson's Disease: Analysis of the PPMI Cohort. Frontiers in neurology. 2019;10:471.

15. Stebbins GT, Goetz CG, Burn DJ, Jankovic J, Khoo TK, Tilley BC. How to identify tremor dominant and postural instability/gait difficulty groups with the movement disorder society unified Parkinson's disease rating scale: Comparison with the unified Parkinson's disease rating scale. Movement Disorders. 2013;28(5):668-70.

16. Shik ML, Severin FV, Orlovskii GN. [Control of walking and running by means of electric stimulation of the midbrain]. Biofizika. 1966;11(4):659-66.

17. Mori S, Sakamoto T, Ohta Y, Takakusaki K, Matsuyama K. Site-specific postural and locomotor changes evoked in awake, freely moving intact cats by stimulating the brainstem. Brain research. 1989;505(1):66-74.

18. Skinner RD, Garcia-Rill E. The mesencephalic locomotor region (MLR) in the rat. Brain research. 1984;323(2):385-9.

19. Eidelberg E, Walden JG, Nguyen LH. Locomotor control in macaque monkeys. Brain : a journal of neurology. 1981;104(Pt 4):647-63.

20. Bernau NA, Puzdrowski RL, Leonard RB. Identification of the midbrain locomotor region and its relation to descending locomotor pathways in the Atlantic stingray, Dasyatis sabina. Brain research. 1991;557(1-2):8394.

21. Cabelguen JM, Bourcier-Lucas C, Dubuc R. Bimodal locomotion elicited by electrical stimulation of the midbrain in the salamander Notophthalmus viridescens. The Journal of neuroscience : the official journal of the Society for Neuroscience. 2003;23(6):2434-9.

22. Musienko PE, Zelenin PV, Lyalka VF, Orlovsky GN, Deliagina TG. Postural performance in decerebrated rabbit. Behavioural brain research. 2008;190(1):124-34.

23. Ryczko D, Dubuc R. The multifunctional mesencephalic locomotor region. Curr Pharm Des. 2013;19(24):444870.

24. Bachmann LC, Matis A, Lindau NT, Felder P, Gullo M, Schwab ME. Deep brain stimulation of the midbrain locomotor region improves paretic hindlimb function after spinal cord injury in rats. Sci Transl Med. 2013;5(208):208ra146.

25. Fluri F, Malzahn U, Homola GA, Schuhmann MK, Kleinschnitz C, Volkmann J. Stimulation of the mesencephalic locomotor region for gait recovery after stroke. Annals of neurology. 2017;82(5):828-40. 
26. Noga BR, Santamaria AJ, Chang S, Benavides FD, Sanchez FJ, Villamil LM, et al. The micropig model of neurosurgery and spinal cord injury in experiments of motor control. In: Whelan PJ, Sharples S, editors. The Neural Control of Movement: Model Systems and Tools to Study Locomotor Function: Academic Press/Elsevier; 2021.

27. Piallat B, Chabardes S, Torres N, Fraix V, Goetz L, Seigneuret E, et al. Gait is associated with an increase in tonic firing of the sub-cuneiform nucleus neurons. Neuroscience. 2009;158(4):1201-5.

28. Tattersall TL, Stratton PG, Coyne TJ, Cook R, Silberstein P, Silburn PA, et al. Imagined gait modulates neuronal network dynamics in the human pedunculopontine nucleus. Nature neuroscience. 2014;17(3):449-54.

29. Jahn K, Deutschlander A, Stephan T, Kalla R, Wiesmann M, Strupp M, et al. Imaging human supraspinal locomotor centers in brainstem and cerebellum. Neuroimage. 2008;39(2):786-92.

30. Follett KA, Torres-Russotto D. Deep brain stimulation of globus pallidus interna, subthalamic nucleus, and pedunculopontine nucleus for Parkinson's disease: Which target? Parkinsonism \& Related Disorders. 2012;18:S165-S7.

31. Weaver FM, Follett K, Stern M, Hur K, Harris C, Marks WJ, Jr., et al. Bilateral deep brain stimulation vs best medical therapy for patients with advanced Parkinson disease: a randomized controlled trial. JAMA. 2009;301(1):63-73.

32. St George RJ, Nutt JG, Burchiel KJ, Horak FB. A meta-regression of the long-term effects of deep brain stimulation on balance and gait in PD. Neurology. 2010;75(14):1292-9.

33. Thevathasan W, Debu B, Aziz T, Bloem BR, Blahak C, Butson C, et al. Pedunculopontine nucleus deep brain stimulation in Parkinson's disease: A clinical review. Mov Disord. 2018;33(1):10-20.

34. Mazzone P, Lozano A, Stanzione P, Galati S, Scarnati E, Peppe A, et al. Implantation of human pedunculopontine nucleus: a safe and clinically relevant target in Parkinson's disease. Neuroreport. 2005;16(17):1877-81.

35. Plaha P, Gill SS. Bilateral deep brain stimulation of the pedunculopontine nucleus for Parkinson's disease. Neuroreport. 2005;16(17):1883-7.

36. Moro E, Hamani C, Poon YY, Al-Khairallah T, Dostrovsky JO, Hutchison WD, et al. Unilateral pedunculopontine stimulation improves falls in Parkinson's disease. Brain : a journal of neurology. 2010;133(Pt 1):215-24.

37. Wang JW, Zhang YQ, Zhang XH, Wang YP, Li JP, Li YJ. Deep Brain Stimulation of Pedunculopontine Nucleus for Postural Instability and Gait Disorder After Parkinson Disease: A Meta-Analysis of Individual Patient Data. World neurosurgery. 2017;102:72-8.

38. Wang H, Gao H, Jiao T, Luo Z. A meta-analysis of the pedunculopontine nucleus deep-brain stimulation effects on Parkinson's disease. Neuroreport. 2016;27(18):1336-44.

39. Golestanirad L, Elahi B, Graham SJ, Das S, Wald LL. Efficacy and Safety of Pedunculopontine Nuclei (PPN) Deep Brain Stimulation in the Treatment of Gait Disorders: A Meta-Analysis of Clinical Studies. The Canadian journal of neurological sciences Le journal canadien des sciences neurologiques. 2016;43(1):120-6.

40. Alam M, Schwabe K, Krauss JK. The pedunculopontine nucleus area: critical evaluation of interspecies differences relevant for its use as a target for deep brain stimulation. Brain : a journal of neurology. 2011;134(Pt 1):11-23.

41. Albin RL, Surmeier DJ, Tubert C, Sarter M, Müller MLTM, Bohnen NI, et al. Targeting the pedunculopontine nucleus in Parkinson's disease: Time to go back to the drawing board. Movement disorders : official journal of the Movement Disorder Society. 2018;33(12):1871-5. 
42. Benarroch EE. Pedunculopontine nucleus: functional organization and clinical implications. Neurology. 2013;80(12):1148-55.

43. Goetz L, Bhattacharjee M, Ferraye MU, Fraix V, Maineri C, Nosko D, et al. Deep Brain Stimulation of the Pedunculopontine Nucleus Area in Parkinson Disease: MRI-Based Anatomoclinical Correlations and Optimal Target. Neurosurgery. 2019;84(2):506-18.

44. Josset N, Roussel M, Lemieux M, Lafrance-Zoubga D, Rastqar A, Bretzner F. Distinct Contributions of Mesencephalic Locomotor Region Nuclei to Locomotor Control in the Freely Behaving Mouse. Curr Biol. 2018;28(6):884-901.e3.

45. Caggiano V, Leiras R, Goñi-Erro H, Masini D, Bellardita C, Bouvier J, et al. Midbrain circuits that set locomotor speed and gait selection. Nature. 2018;553(7689):455-60.

46. Chang SJ, Cajigas I, Opris I, Guest JD, Noga BR. Dissecting brainstem locomotor circuits: converging evidence for cuneiform nucleus stimulation. Frontiers in systems neuroscience. 2020.

47. Opris I, Dai X, Johnson DMG, Sanchez FJ, Villamil LM, Xie S, et al. Activation of Brainstem Neurons During Mesencephalic Locomotor Region-Evoked Locomotion in the Cat. Frontiers in systems neuroscience. 2019;13:69.

48. Zitella LM, Mohsenian K, Pahwa M, Gloeckner C, Johnson MD. Computational modeling of pedunculopontine nucleus deep brain stimulation. Journal of neural engineering. 2013;10(4):045005.

49. Giannini G, Francois M, Lhommée E, Polosan M, Schmitt E, Fraix V, et al. Suicide and suicide attempts after subthalamic nucleus stimulation in Parkinson disease. Neurology. 2019;93(1):e97-e105.

50. Cordeiro JG, Diaz A, Davis JK, Di Luca DG, Farooq G, Luca CC, et al. Safety of Noncontrast Imaging-Guided Deep Brain Stimulation Electrode Placement in Parkinson Disease. World neurosurgery. 2020;134:e1008-e14.

51. Ferraye MU, Debu B, Fraix V, Goetz L, Ardouin C, Yelnik J, et al. Effects of pedunculopontine nucleus area stimulation on gait disorders in Parkinson's disease. Brain : a journal of neurology. 2010;133(Pt 1):205-14.

52. Horn A, Kühn AA. Lead-DBS: A toolbox for deep brain stimulation electrode localizations and visualizations. Neurolmage. 2015;107:127-35.

53. Olszewski J, Baxter D. Cytoarchitecture of the human brain stem. Basel: S. Karger; 1982.

\section{Figures}




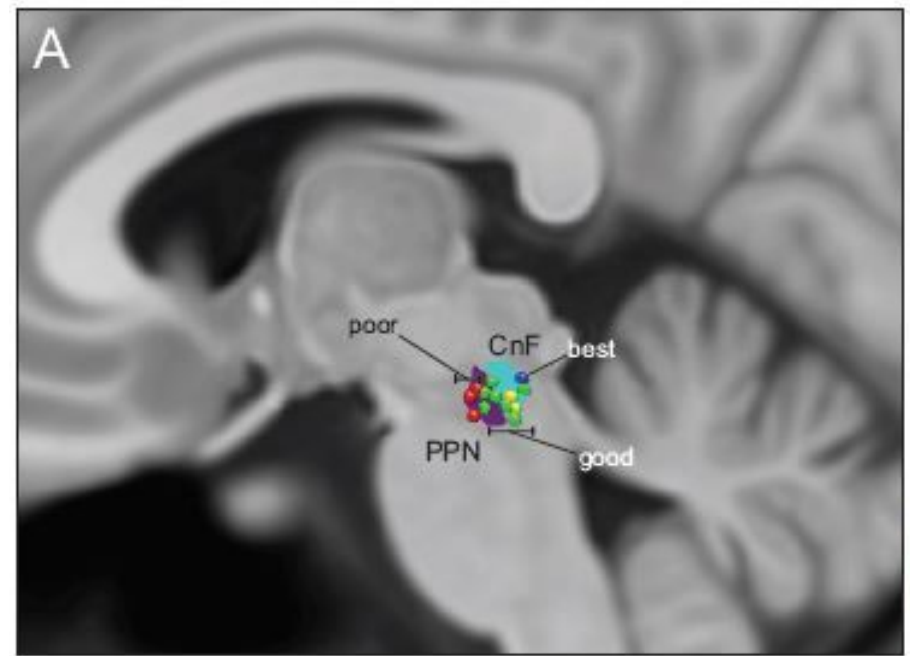

C

\begin{tabular}{|c|c|c|}
\hline Structure & Relation to $\mathrm{CnF}$ & Effect of Stimulation \\
\hline Periaqueductal gray (not shown) & Darsomedial & Anxiety, aversion, discomfort \\
\hline Mesencephalic nucleus of trigeminal nerve (not shown) & Medial & Jaw movement \\
\hline Locus coeruleus (LC) & Medial, deep & Possible changes in alertness, arousal \\
\hline Medial longitudinal fasciculus (not shown) & Medial & Ocular deviation, diplapia, oscillopsia \\
\hline Trochlear nucleus (not shown) & Medial & Ocular deviation, diplopia, oscillopsia \\
\hline Ducussation of superior cerebellar peduncle (dSCP) & MediaVDorsomedial & Nystagmus, vertigo, ataxia \\
\hline Central tegmental tract (CTT) & Medial & Palatal movements \\
\hline Medial lemniscus (ML) & LateralNentrolateral & Paresthesias \\
\hline Spinothalamic tract (STT) & Lateral/Dorsolateral & Pain and temperature sensations \\
\hline Lateral lemniscus (not shown) & Lateral & Auditory phenomena, buzzing \\
\hline
\end{tabular}

\section{Figure 1}

Three-dimensional anatomy of the human MLR and expected side effects of DBS. Reconstructions were made using Lead-DBS and available MNI-space subcortical atlases.(52) A separate CnF (cyan)NIfTI object was created in relation to the PPN (dark purple) based on Olszewski and Baxter's Atlas (53).(A) Sagittal projection (5mm lateral to midline) of the $\mathrm{CnF}$ (cyan) and PPN (dark purple) with overlay of active contacts from PPN DBS patients with poor (red), good (green), best (blue), and unevaluated (yellow) gait outcomes from Goetz et al.(43)(B)Diagonal 3D view with right $M L$ and STT absent to visualize the MLR, projected on to a transverse slice of the brain at the level of the pons. (C)Chart lists nearby structures, their relation to the $\mathrm{CnF}$, and expected side effects of stimulation. $\mathrm{CnF}$ Cuneiform nucleus, CTT central tegmental tract, dSCP decussation of superior cerebellar peduncle, LC locus coeruleus, ML medial lemniscus, PPN Pedunculopontine nucleus, RN red nucleus, STT spinothalamic tract.Adapted from Fig. 2 of (46). 


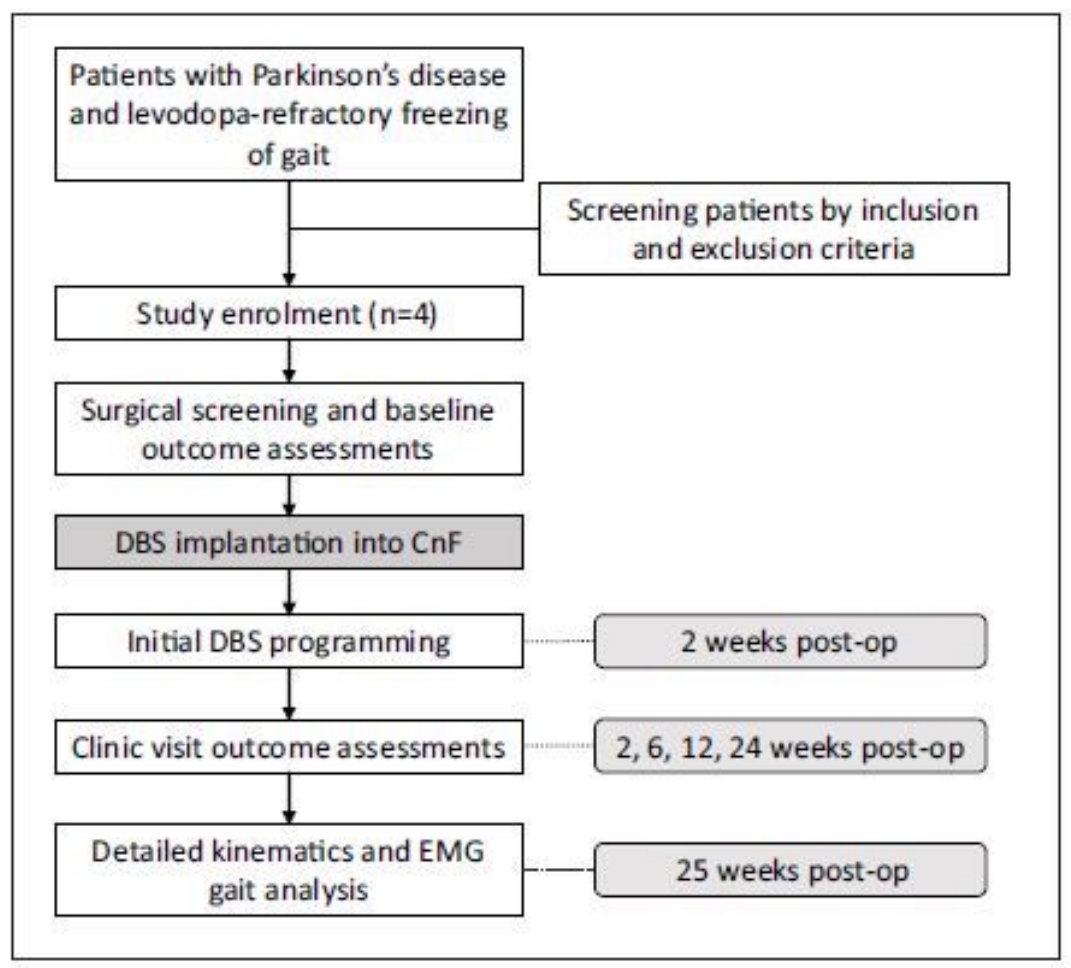

Figure 2

Flow chart of study outline

\section{Supplementary Files}

This is a list of supplementary files associated with this preprint. Click to download.

- SPIRITchecklist.doc 